\title{
Non-Markovian Dissipative Semiclassical Dynamics
}

\author{
Werner Koch, ${ }^{1}$ Frank Großmann, ${ }^{1}$ Jürgen T. Stockburger, ${ }^{2}$ and Joachim Ankerhold ${ }^{2}$ \\ ${ }^{1}$ Institut für Theoretische Physik, Technische Universität Dresden, D-01062 Dresden, Germany \\ ${ }^{2}$ Institut für Theoretische Physik, Universität Ulm, D-89069 Ulm, Germany
}

\begin{abstract}
The exact stochastic decomposition of non-Markovian dissipative quantum dynamics is combined with the time-dependent semiclassical initial value formalism. It is shown that even in the challenging regime of moderate friction and low temperatures, where non-Markovian effects are substantial, this approach allows for the accurate description of dissipative dynamics in anharmonic potentials over many oscillation periods until thermalization is reached. The problem of convergence of the stochastic average at long times, which plagues full quantum mechanical implementations, is avoided through a joint sampling of the stochastic noise and the semiclassical phase space distribution.
\end{abstract}

PACS numbers: 03.65.Yz, 05.40.-a 82.20.-w

The understanding of the nonequilibrium dynamics of open quantum systems has been a central challenge in the last decades [1, 2]. In recent years the subject has gained considerable interest due to experimental progress which allows for the tailoring and manipulation of quantum matter on ever larger scales. In mesoscopic physics, for instance, superconducting circuits have been realized to observe coherent dynamics and entanglement [3]. Similar advance has been achieved on molecular scales with the detection of interferences in wave packet dynamics and the control of the population of specific molecular states [4]. Typically, these systems are in contact with a large number of environmental degrees of freedom, e. g. electromagnetic modes of the circuitry or residual vibronic modes, which give rise to decoherence and relaxation [5]. In many cases, the idealization of an isolated system must inevitably be replaced by an open-system theory.

In the standard approach to open quantum systems, the reduced dynamics of the system of interest is obtained by tracing out "reservoir" degrees of freedom from the conservative system-plus-reservoir dynamics, e. g., through projection operator techniques. Alternatively, this program can be carried out through exact path integral expressions for the reduced density matrix [6], which became widely used in the 1980s [1]. The distinguishing feature of dissipative path integrals is an influence functional which describes self-interactions non-local in time. Hence, a simple quantum mechanical analogue to the classical Langevin equation is not known; commonly used equations, such as Master/Redfield equations [2] in the weak-coupling case and quantum Smoluchowski equations [7] for reservoir-dominated dynamics, rely on perturbation theory. In intermediate domains, quantum Monte Carlo techniques have been put forward for tightbinding systems, but the achievable propagation times are severely limited by the dynamical sign problem.

Recently, it has been shown that the influence functional can be exactly reproduced through stochastic averaging of a process without explicit memory [8, 9]. The formulation turned out to be particularly efficient for weak to moderate friction and low temperatures [8, 10], a regime which lies beyond the strict validity of Redfield equations on the one hand and beyond the applicability of Monte Carlo schemes on the other hand. The drawback is though that for nonlinear systems the convergence of the stochastic average for relatively long times is still an unsolved problem. Some progress has been made for the spin-boson model by using a hierarchical approach to quantum memory terms [11]. A reliable and efficient, generally applicable method to tackle the dissipative dynamics for continuous systems in this challenging parameter regime is still missing.

Here we address this issue by combining the exact stochastic Schrödinger formulation with a semiclassical real-time technique based on a time-dependent initial value representation of the quantum mechanical propagator. The central finding is that this procedure circumvents the main obstacle of the exact formulation and allows for accurate simulations up to times where equilibration sets in. It is important to note that a direct stationary-phase evaluation of the double path integral for the reduced density is not a consistent semiclassical approximation since the classical limit of open-system dynamics, the Langevin equation, is not recovered [1, 12].

Undamped systems allow a powerful method based on the semiclassical propagator of Herman and Kluk (HK) [13], which has seen an impressive number of applications ranging from atomic [14 to chemical physics 15] after the work of Kay [16] stimulated renewed interest in the approach. The HK propagator was recently shown to be the leading term of consistent series expansions of the exact quantum propagator of a Hamiltonian system [17, 18]. Currently, efforts are under way to directly extend this approach to dynamics with quantum memory effects [19]. In this Letter, we will use a memory-free representation, which accounts for non-Markovian dynamics entirely through correlations of complex noise forces and thus suits numerical applications.

We start with the standard decomposition of the total Hamiltonian

$$
\hat{H}=\hat{H}_{S}+\hat{H}_{B}+\hat{H}_{I}
$$


as a sum of a system part, that for reasons of simplicity shall here depend on one degree of freedom $x$, a bath part consisting of an infinity of harmonic oscillators together with a bilinear interaction between them. In case of a factorized initial density with a bath residing in thermal equilibrium at temperature $T$ one derives a path integral expression for the time-evolved reduced density matrix of the form [1]

$$
\begin{aligned}
\rho\left(x_{f}, x_{f}^{\prime}, t\right)= & \int d x_{i} d x_{i}^{\prime} \rho\left(x_{i}, x_{i}^{\prime}, 0\right) \int \mathcal{D}\left[x_{1}\right] \mathcal{D}\left[x_{2}\right] \\
& \exp \left\{\frac{i}{\hbar}\left(S_{\mathrm{S}}\left[x_{1}\right]-S_{\mathrm{S}}\left[x_{2}\right]\right)\right\} F\left[x_{1}, x_{2}\right],(2)
\end{aligned}
$$

where the two real time paths $x_{1}$ and $x_{2}$ run in time $t$ from $x_{i}$ and $x_{i}^{\prime}$ to $x_{f}$ and $x_{f}^{\prime}$, respectively. They are coupled by the influence functional, which takes the form $F[y, r]=\exp (-\Phi[y, r] / \hbar)$ with

$$
\begin{aligned}
\Phi[y, r]= & \frac{1}{\hbar} \int_{0}^{t} d u \int_{0}^{u} d v y(u)\left[L^{\prime}(u-v) y(v)+2 i L^{\prime \prime}(u-v) r(v)\right. \\
& +i \mu \int_{0}^{t} d u y(u) r(u)
\end{aligned}
$$

where $y=x_{1}-x_{2}, r=\left(x_{1}+x_{2}\right) / 2$ denote difference and sum paths, respectively. The complex valued friction kernel $L(t)=L^{\prime}(t)+i L^{\prime \prime}(t)$ is related to the force-force auto-correlation function of the bath and completely determined by its spectral density $J(\omega)$ and inverse temperature $\beta$. The static susceptibility denoted by $\mu=-\int_{0}^{\infty} d u L^{\prime \prime}(u) /(2 \hbar)$ is a property of the reservoir.

In [9] it has been shown that a stochastic unraveling of the forward and the backward paths leads to

$$
\begin{aligned}
\rho\left(x_{f}, x_{f}^{\prime}, t\right) & =\int d x_{i} \int d x_{i}^{\prime} \rho\left(x_{i}, x_{i}^{\prime}, 0\right) \\
\times & M\left[K_{z_{1}}\left(x_{f}, t ; x_{i}, 0\right)\left(K_{z_{2}}\left(x_{f}^{\prime}, t ; x_{i}^{\prime}, 0\right)\right)^{*}\right],
\end{aligned}
$$

where $M$ denotes the average over noise realizations $z_{j}$ $(j=1,2)$, with the noise augmenting the system actions via

$$
S_{z_{j}}\left[x_{j}\right]=S_{S}\left[x_{j}\right]+\mu \int_{0}^{t} d u x_{j}(u)^{2}+\int_{0}^{t} d u x_{j}(u) z_{j}(u)
$$

in the path integral expressions of the respective propagators $K_{z_{j}}$. This stochastic unraveling differs from a similar one by Strunz et al. [20] through the appearance of two noise variables, allowing for the elimination of quantum memory effects.

Representing a general initial density operator through $\hat{\rho}(t=0)=\left|\Psi_{1}\right\rangle\left\langle\Psi_{2}\right|$ (or through an ensemble of such projectors) leads to two Schrödinger equations

$$
\begin{aligned}
i \hbar\left|\dot{\Psi}_{1}\right\rangle & =\left[H_{\mathrm{S}}-\xi(t) x+\frac{\mu}{2} x^{2}-\frac{\hbar}{2} \nu(t) x\right]\left|\Psi_{1}\right\rangle \\
i \hbar\left|\dot{\Psi}_{2}\right\rangle & =\left[H_{\mathrm{S}}-\xi^{*}(t) x+\frac{\mu}{2} x^{2}+\frac{\hbar}{2} \nu^{*}(t) x\right]\left|\Psi_{2}\right\rangle,
\end{aligned}
$$

where $\xi(t)=\frac{1}{2}\left[z_{1}(t)+z_{2}^{*}(t)\right]$ and $\nu(t)=\frac{1}{\hbar}\left[z_{1}(t)-z_{2}^{*}(t)\right]$. The reduced density matrix (2) is reproduced exactly by averaging $\hat{\rho}$ obtained from equations (6) and (7) when the correlations of $\xi$ and $\nu$ reproduce the integral kernel of the influence functional: $M\left[\xi(t) \xi\left(t^{\prime}\right)\right]=$ $L^{\prime}\left(t-t^{\prime}\right), M\left[\xi(t) \nu\left(t^{\prime}\right)\right]=(2 i / \hbar) \Theta\left(t-t^{\prime}\right) L^{\prime \prime}\left(t-t^{\prime}\right)$, and $M\left[\nu(t) \nu\left(t^{\prime}\right)\right]=0$ ( $\Theta$ denotes the Heaviside step function).

The linear equations (6) and (7) are formally exact in the sense that their Monte Carlo sampling will eventually converge to yield Eq. (2). However, they are of limited use for practical calculations since individual samples do not stay normalized, which slows down convergence and makes a direct samping impractical [2, 9]. This slowdown can be reduced [10] by an exact mapping of the stochastic processes (6) and (7) to a trace-conserving process (similar to a Girsanov transform [21]). Observing that only the last terms in the square brackets of equations (6) and (7) lead to a change of tr $\hat{\rho}$, the first step of the transform consists of subtracting an arbitrary "reference )] trajectory" $\bar{r}_{u}$ from $x$ in these terms [22]. The effect of this modification is canceled exactly by a corresponding (3) change in the probability measure, which can be represented by the substitution

$$
\xi \rightarrow \tilde{\xi}=\xi-\int_{0}^{t} d u \chi(t-u) \bar{r}_{u}
$$

where $\chi(u)=-\Theta(u) L^{\prime \prime}(u) / 2 \hbar$ is the response function of the reservoir. Details of the transform are given in Refs. 9] and [10]. With $\bar{r}_{u}=\left\langle\Psi_{1}|x| \Psi_{2}\right\rangle_{u}$, the diffusion of $\operatorname{tr} \hat{\rho}$ is eliminated. However, this can lead to subtle mathematical difficulties limiting the times for which numerical simulations are stable [10]. There are two situations known to be free of such instabilities, namely, linear systems and the classical limit. The idea is thus to combine the stochastic quantum dynamics with a semiclassical propagation scheme based on the frozen Gaussian approximation pioneered by Herman and Kluk [13] and Heller [23].

The propagation of individual samples of the stochastic processes (6) and (77) by the HK propagator differs from the evolution of a closed system only through the addition of simple potential terms, up to quadratic order, to the system Hamiltonian. Hence the asymptotic convergence properties of the HK propagator for closed systems [18, 24, 25] are 'inherited' by our stochastic samples.

The semiclassical HK propagator is given in terms of a phase space integral as

$$
\begin{aligned}
K\left(x_{f}, t, x_{i}, 0\right) & =\int \frac{d p_{i} d q_{i}}{2 \pi \hbar}\left\langle x_{f} \mid g_{\gamma}\left(p_{t}, q_{t}\right)\right\rangle \\
& \times R\left(p_{i}, q_{i}, t\right) \mathrm{e}^{i S\left(q_{i}, p_{i}, t\right) / \hbar}\left\langle g_{\gamma}\left(p_{i}, q_{i}\right) \mid x_{i}\right\rangle .
\end{aligned}
$$

Here complex valued Gaussian wave packets $\left\langle x \mid g_{\gamma}\right\rangle \sim$ $\exp \left\{-\frac{\gamma}{2}(x-q)^{2}+\frac{i}{\hbar} p(x-q)\right\}$ of fixed width parameter $\gamma$ have been introduced, centered around the initial phase space points $p_{i}, q_{i}$ and the time-evolved phase space 
points $p_{t}, q_{t}$, respectively. The pre-exponential factor $R$ contains a complex valued combination of stability matrix elements and the action reads as in (5) with the replacement of the noise force described in equation (8). Obtaining the final density matrix $\rho\left(x_{f}, x_{f}^{\prime}, t\right)$ involves three Monte Carlo integrations, two over the forward and backward phase spaces of the semiclassical propagators and an additional one over the noise trajectory distribution.

The classical trajectory entering equation (9) is obtained from the quasiclassical dynamics of the fixedwidth Gaussians under the transformed versions of equations (6) and (7). The complex forces $\xi$ and $\nu$ do not extend the phase space to complex numbers: The frozen Gaussians are, up to a trivial phase factor, coherent states $|\alpha\rangle=e^{-\left|\alpha^{2}\right| / 2} e^{\alpha \hat{a}^{\dagger}}|0\rangle$ with

$$
\alpha=\sqrt{\frac{\gamma}{2}}\left(q+\frac{i p}{\hbar \gamma}\right)
$$

It is clear from this equation that complex values of $q$ and $p$ lead only to states already described by a real-valued phase space. In compact form, the classical equations of motion derived from (6) and (7) read $(j=1,2)$

$$
\frac{d}{d t} \alpha_{j}=\sqrt{\frac{\gamma}{2}}\left(\frac{p_{j}}{m}-\frac{i}{\hbar \gamma} V^{\prime}\left(q_{j}\right)+\frac{i}{\hbar \gamma} f_{j}\right)
$$

with $f_{1}=\tilde{\xi}+\frac{\hbar}{2} \nu$ and $f_{2}=\tilde{\xi}^{*}-\frac{\hbar}{2} \nu^{*}$, to be solved for real $q_{j}$ and $p_{j}$. Taking the limit $\hbar \rightarrow 0$, and integrating by parts in equation (8), the classical Langevin equation is indeed recovered from equation (11).

In the semiclassical context, the reference trajectory is again obtained by demanding that the $\nu$-dependent terms in equations (6) and (7) do not change the trace of the sample, which leads to the simple condition

$$
\bar{r}_{u}=\left(\alpha_{1}+\alpha_{2}^{*}\right) / \sqrt{2 \gamma} .
$$

This definition of $\bar{r}_{u}$ makes reference to a single pair of semiclassical trajectories. It is therefore advantageous to merge the integrations over the two HK phase spaces and the function space of noise trajectories $\xi(t)$ and $\nu(t)$ into a joint Monte Carlo sampling scheme where new initial phase space points and a new noise trajectory are drawn for each sample [26].

To demonstrate the approach, in the remainder we will concentrate on the dynamics in a one-dimensional Morse potential

$$
V(x)=\hbar D\left\{1-\exp \left[-a\left(x-x_{0}\right) / x_{0}\right]\right\}^{2},
$$

which may serve e. g. as a simple model for the relative motion of a diatomic molecule with equilibrium separation $x_{0}$. The HK propagation for the isolated system is known to accurately reproduce the exact quantum dynamics. Here we introduce the scaled displacement

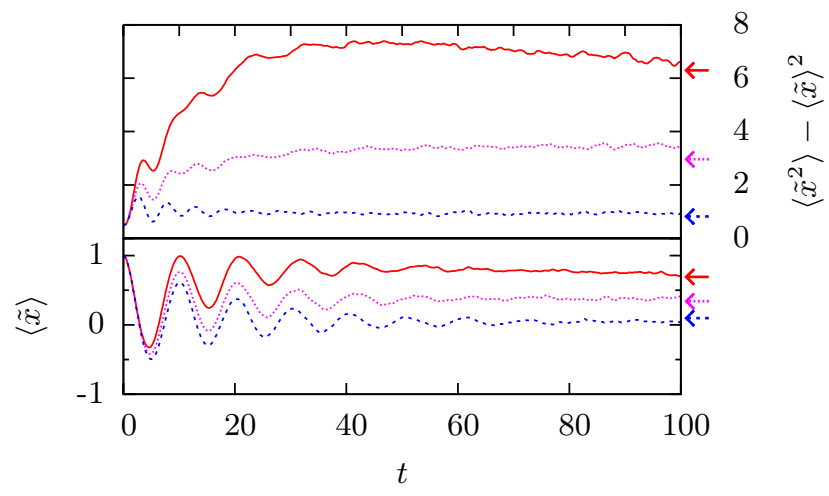

FIG. 1: Expectation value of position and variance in a dissipative Morse oscillator for decreasing temperature $\hbar \beta=0.5$ (full), $\hbar \beta=1$ (dotted), $\hbar \beta=10$ (dashed) at constant $\eta=0.1$. Arrows indicate thermal expectation values of the unperturbed oscillator (see text).

$\tilde{x}=\left(x-x_{0}\right) / x_{0}$ and the frequency unit $\hbar /\left(m x_{0}^{2}\right)$. In the following we use the parameters $D=30$ and $a=0.08$ corresponding to a total number of 97 bound states. In these units the frequency $\omega_{0}$ for small oscillations around the well minimum located at $\tilde{x}=0$ is $\omega_{0}=a \sqrt{2 D}=0.62$. In the Markovian case and with the restriction to the bound state part of the spectrum a similar system has been studied in [27]. As the initial state of the propagation we have chosen a Gaussian wave packet shifted away from the potential minimum with $\langle\tilde{x}\rangle_{0}=1$ and zero initial momentum. The spectral density of the bath oscillators is taken to be Ohmic with an algebraic cutoff

$$
J(\omega)=\frac{\eta \omega}{\left(1+\omega^{2} / \omega_{c}^{2}\right)^{2}},
$$

where the cutoff frequency $\omega_{c}=10$ is well beyond any relevant system frequencies and the dimensionless coupling strength is denoted by $\eta$.

Let us first look at the temperature dependence of mean and variance in position for a fixed interaction strength $\eta=0.1$, displayed in Fig. 1. As expected, damped oscillations occur for intermediate times. In sharp contrast to the full quantum mechanical case, however, the simulations are stable over long periods of time until equilibration is approached. Arrows indicate equilibrium values obtained by Boltzmann weighting analytic bound-state results from [28]. At higher temperatures the mean saturates at a value considerably away from zero thus revealing the substantial influence of the anharmonicity. This influence gradually decreases when the temperature is lowered. Only at the lowest temperature $(\hbar \beta=10$, dashed arrows) are the results consistent with a harmonic approximation of the oscillator. Similar effects are seen in the position variance, with the additional feature that coherent oscillations are strongly smeared out at higher temperatures. Importantly, for the lowest temperatures considered here, one has $\omega_{c} \hbar \beta \gg 1$ so that retardation effects are strong and the dynamics 


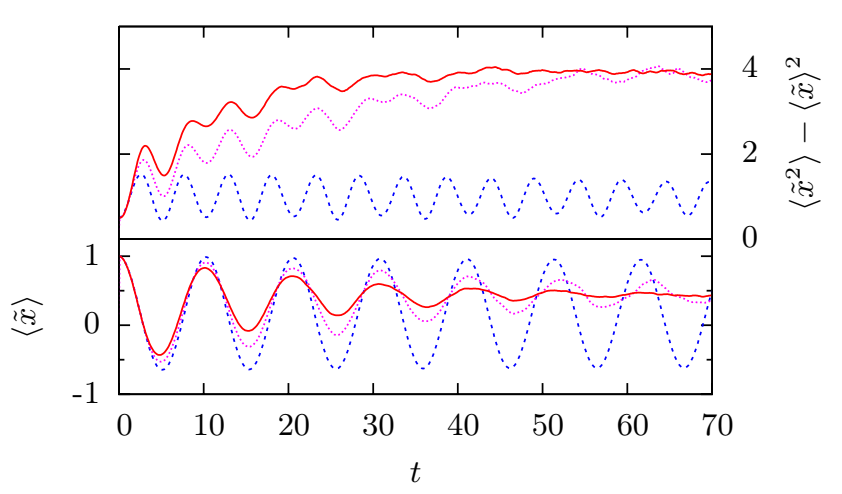

FIG. 2: Same as in Fig. 1, but for increasing coupling $\eta=0$ (dashed), $\eta=0.05$ (dotted), $\eta=0.1$ (full), all at $\hbar \beta=1$.

is far from being Markovian. Further, since $\eta \hbar \beta \approx 1$ we are outside the weak coupling regime in the sense of Master/Redfield equations. To check the thermal nature of the final expectation values, we used different initial preparations and found that the results converge to the same long time average (not shown). The total number of sampling points needed to generate all presented results is $10^{7}$; the calculations take a few hours on a desktop PC.

In Figure 2 the same expectation values are depicted at a fixed inverse temperature $\hbar \beta=1$ but varying dissipation strengths. Of course, pronounced coherent oscillations are seen for vanishing coupling, where again the anharmonicity of the potential shows up in smaller oscillation amplitudes for negative than for positive values of position. Now a marked difference between the isolated system and the case of weak damping can be observed: Even for weak damping the amplitudes of the oscillations decrease strongly, with centers shifted towards the soft side of the potential. The variance increases as a function of time, while its initial oscillations decay on a somewhat shorter timescale. We mention that at the fixed temperature $\hbar \beta=1$, almost identical long time averages are reached for all small friction strengths that we have investigated.

To summarize, we have introduced a well-defined semiclassical propagator for non-Markovian open quantum systems. It was derived by consistently applying a known stochastic decomposition of the influence functional appearing in exact open-system path integrals. A test using a standard model of molecular physics shows that the dissipative semiclassical propagator accurately describes the time evolution over long periods of time, up to thermal equilibration between open system and environment. With modest computational resources, we provide data for this model which are difficult if not impossible to obtain by other means in the low-temperature regime of non-Markovian dynamics. For systems where corrections to the HK propagator are needed, extensions according to [18] are straightforward. We estimate that the number of samples needed for a higher-dimensional quantum system will not increase substantially beyond the number of samples in the one-dimensional case because the phase-space sampling converges more rapidly than the noise average.

W. K., F. G., and J. T. S. acknowledge financial support by the Deutsche Forschungsgemeinschaft (GR 1210/4-1, SFB 382) and J. A. by the German-Israeli foundation.

[1] U. Weiss, Quantum Dissipative Systems, 2nd ed. (World Scientific, Singapore, 1999).

[2] H.-P. Breuer, F. Petruccione, The Theory of Open Quantum Systems (Oxford University Press, Oxford, 2002).

[3] G. Wendin, V.S. Shumeiko, Low Temp. Phys. 33, 724 (2007).

[4] C. M. Tesch, R. de Vivie-Riedle, Phys. Rev. Lett. 89, 157901 (2002).

[5] W. H. Zurek, Phys. Today 44, 36 (1991).

[6] R. P. Feynman and F. L. Vernon, Ann. Phys. (N. Y.) 24, 118 (1963).

[7] J. Ankerhold, P. Pechukas, H. Grabert, Phys. Rev. Lett. 87, 086802 (2001).

[8] J. T. Stockburger, H. Grabert, Chem. Phys. 268, 249 (2001).

[9] J. T. Stockburger, H. Grabert, Phys. Rev. Lett. 88, 170407 (2002).

[10] J. T. Stockburger, Chem. Phys. 296, 159 (2004).

[11] Y. Yan, F. Yang, Y. Liu, J. Shao, Chem. Phys. Lett. 395, 216 (2004).

[12] F. Grossmann, J. Chem. Phys. 103, 3696 (1995).

[13] M. F. Herman, E. Kluk, Chem. Phys. 91, 27 (1984).

[14] G. van de Sand, J.-M. Rost, Phys. Rev. Lett. 83, 524 (1999).

[15] M. Thoss, H. Wang, Ann. Rev. Phys. Chem. 55, 299 (2004).

[16] K. G. Kay, J. Chem. Phys. 100, 4377 (1994); ibid 100, 4432 (1994); ibid 101, 2250 (1994).

[17] S. Zhang, E. Pollak, Phys. Rev. Lett. 91, 190201 (2003).

[18] K. Kay, Chem. Phys. 322, 3 (2006); G. Hochman and K. G. Kay, Phys. Rev. A 73, 064102 (2006).

[19] E. Pollak, J. Chem. Phys. 127, 074505 (2007).

[20] W.T. Strunz, L. Diósi, N. Gisin, Phys. Rev. Lett. 82, 1801 (1999).

[21] D. Gatarek, N. Gisin, J. Math. Phys. 32, 2152 (1991).

[22] The reference trajectory may be any causal, analytic functional of $\xi$ and $\nu$.

[23] E. J. Heller, J. Chem. Phys. 75, 2923 (1981).

[24] E. Martín-Fierro and J. M. Gomez Llorente, Chem. Phys. 322, 13 (2006).

[25] T. Swart and V. Rousse, arXiv:0712.0752 v2 [math-ph]

[26] Since the initial HK phase space density and the noise probability are independent, other sampling schemes are possible, but the close relation of equation (11) to the classical Langevin equation may be lost.

[27] H.-P. Breuer, W. Huber, F. Petruccione, Comp. Phys. Comm. 104, 46 (1997).

[28] J. A. C. Gallas, Phys. Rev. A 21, 1829 (1980). 